\title{
Recessões e recuperações na atividade econômica: mesmo efeito sobre a criminalidade?
}

\author{
Ari Francisco de Araujo Junior* \\ Cláudio Djissey Shikida*
}

\begin{abstract}
RESUMO - Evidências sobre a economia do crime mostram que o ambiente socioeconômico é um importante determinante das atividades criminosas. Este artigo se baseia na hipótese de que existem assimetrias na criminalidade conforme as fases do ciclo econômico segundo Mocan \& Bali (2007). Por meio dos dados do Índice Mineiro de Responsabilidade Social (IRMS) foi estimado um painel dinâmico e encontrou-se que, para crimes contra o patrimônio, existem evidências de assimetria no ciclo econômico de forma que aumentos no desemprego municipal geram um aumento no crime municipal menor que as quedas no mesmo quando da redução do desemprego. Em outras palavras, criminosos envolvidos em crimes contra o patrimônio têm mais facilidade a voltar ao mercado de trabalho legal.
\end{abstract}

Palavras-chave: Economia do Crime. Assimetria. Inércia Criminal.

\section{INTRODUÇÃO}

Modelos com enfoque microeconômico, em geral, sugerem que os criminosos potenciais atribuem um valor monetário aos ganhos da atividade criminosa e, racionalmente, comparam este valor ao custo monetário relacionado à sua realização. Aqui estariam incluídos o custo de planejamento e execução, outros custos de oportunidade, o custo esperado de serem detidos e condenados e, ainda, um custo moral atribuído ao ato de desrespeitar a lei (BECKER, 1968).

Do ponto de vista empírico, os fatores ou incentivos que captam os benefícios e custos e exercem influência nas taxas de crime são amplamente conhecidos. Tais incentivos estão relacionados, fundamentalmente, ao ambiente econômico, a aspectos sociais e de background familiar, a fatores demográficos e ao aparato de segurança ${ }^{1}$. Alguns trabalhos de natureza econométrica foram realizados para o Brasil e, de modo geral, corroboram o esquema teórico aci$\mathrm{ma}^{2}$. Seja a partir de modelos microestáticos ou dinâmicos, a hipótese subjacente dos modelos teóricos que balizam os estudos empíricos é a de que a magnitude do efeito dos determinantes é constante ou simétrico, o que significa que os impactos de variações nas oportunidades no mercado de trabalho, por exemplo, seriam iguais em períodos de recuperação ou recessão.

\footnotetext{
* Mestre em teoria econômica pela Universidade Federal de Minas Gerais. É professor do Ibmec Minas.

** Doutor em economia pela Universidade Federal do Rio Grande do Sul. É professor titular do Ibmec Minas.

1 Para uma análise dos determinantes das taxas de crime ver, por exemplo, Fajnzylber \& Araujo Junior (2001).

2 Ver, por exemplo, Santos (2009).
} 
Modelos teóricos mais recentes, tal como Mocan, Billups \& Overland (2005), sugerem que a resposta da violência a mudanças na atividade econômica e no aparato de segurança pode ser assimétrica. Por exemplo, o capital humano legal de uma pessoa sofre uma perda enquanto o capital humano criminal aprecia caso ela entre no mundo do crime. É esperada uma dificuldade maior para a volta ao setor legal (ou para reduzir o tempo gasto no crime) caso o engajamento na atividade criminal tenha ocorrido durante uma recessão. Neste caso, pode-se esperar a presença de histerese criminal devido às decisões dinâmicas individuais (relacionadas à maximização de utilidade) entre trabalho no setor criminal ou no setor legal, de tal forma que a redução da criminalidade durante os períodos de recuperação na atividade econômica sejam menores que os aumentos observados durante as recessões. Mocan \& Bali (2007) sugerem metodologia para testar a presença de efeitos assimétricos das mudanças nos determinantes sobre a criminalidade. O objetivo do artigo é realizar um exercício tal como proposto por Mocan \& Bali (2007) para os municípios de Minas Gerais para o período de 2000 a 2007. Foi possível, ainda, medir a magnitude da inércia criminal.

\section{METODOLOGIA}

Como afirmam Mocan \& Bali (2007), normalmente o efeito das oportunidades no mercado de trabalho sobre as taxas de crime é estimado da seguinte forma:

$$
C R=\alpha_{0}+\alpha_{1} L M O+\varepsilon
$$

Em que $\mathrm{CR}=$ taxa de crime, $\mathrm{LMO}=$ oportunidades no mercado de trabalho (medidas, por exemplo, pela taxa de desemprego); $\varepsilon=$ erro; $\alpha_{0}$ e $\alpha_{1}$ são os parâmetros a serem estimados.

Caso a proxy para LMO seja a taxa de desemprego e, de acordo com os vários modelos econômicos dos determinantes do comportamento criminoso, espera-se que $\alpha_{1}$ seja positivo, ou seja, quanto maior a taxa de desemprego maior será a taxa de crime. Vale ressaltar que, neste caso, a hipótese subjacente é a de que a magnitude do efeito é constante ou simétrica, o que significa que os impactos de variações nas oportunidades no mercado de trabalho seriam iguais em períodos de recuperação ou recessão.

Baseado em um modelo teórico proposto em Mocan, Billups \& Overland (2005), Mocan \& Bali (2007) sugerem uma abordagem paramétrica que explora a característica de painel dos dados para testar a existência de assimetria dos efeitos descritos anteriormente. A equação a ser estimada pelos autores foi a seguinte: 


$$
C R_{i t}=\alpha_{0}+\beta L M O^{+}{ }_{i t}+\gamma L M O^{-}{ }_{i t}+X^{\prime} \Omega+\mu_{i}+\Psi_{t}^{\prime}+\Gamma_{i t}+\varepsilon_{i t}
$$

Em que $\mathrm{CR}_{\mathrm{it}}=$ taxa de crime na unidade i e período t; $\mathrm{LMO}^{+}{ }_{\mathrm{it}}=$ aumento do desemprego na unidade i e período t em relação ao período anterior (períodos de recessão); $\mathrm{LMO}^{-}{ }_{\text {it }}=$ aumento no desemprego na unidade i e período t em relação ao período anterior (períodos de recuperação); $\mathrm{X}_{\mathrm{it}}=$ vetor de características das unidades; $\mu_{\mathrm{i}}=$ atributo não observado da unidade de análise que influência o crime; $\Psi_{\mathrm{t}}=$ efeito período; $\Gamma_{\mathrm{it}}=$ tendência temporal específica da unidade i; $\varepsilon_{\mathrm{it}}=$ erro; $\alpha_{0}, \beta$, $\gamma$ e $\Omega=$ conjunto de parâmetros a serem estimados.

As variáveis $\mathrm{LMO}^{+}{ }_{\text {it }}$ e $\mathrm{LMO}^{+}$it são construídas da seguinte forma:

$$
\begin{aligned}
& L M O^{+}{ }_{i \neq t}=\left\{\begin{array}{l}
L M O_{i, t} \text { se } L M O_{i, t} \geq L M O_{i, t-1} \\
0 \text { se } L M O_{i, t}<L M O_{i, t-1}
\end{array}\right. \\
& L M O^{-}{ }_{i, t}=\left\{\begin{array}{l}
L M O_{i, t} \text { se } L M O_{i, t}<L M O_{i, t-1} \\
0 \text { se } L M O_{i, t} \geq L M O_{i, t-1}
\end{array}\right.
\end{aligned}
$$

Acreditamos que a proposta de estimação que consta em Mocan \& Bali (2007) é inadequada neste caso. Utilizaremos aqui o estimador de GMM (Generalized Method of Moments) tal como proposto por Holtz-Eakin, Newey \& Rosen (1990), Arellano \& Bond (1991) e Arellano \& Bover (1995) já que: (1) algumas variáveis explicativas são potencialmente endógenas (devido a problemas de simultaneidade ou causalidade reversa) à violência, como as variáveis de deterrence e (2) a mais correta especificação, neste caso, sugere a presença de efeitos fixos não observados de período e de municípios num modelo de regressão em painel com características dinâmicas (inclui-se na especificação, desta forma, inércia criminal). A consistência do estimador de GMM depende da validade dos valores defasados das variáveis explicativas como instrumentos na regressão dos determinantes da violência. Para tanto utilizamos testes de sobreidentificação de restrições de Sargan e de correlação serial como sugerido por Arellano \& Bond (1991) e Arellano \& Bover (1995).

As informações utilizadas neste artigo constam da base de dados IMRS (Índice Mineiro de Responsabilidade Social) compilada pela Fundação João Pinheiro. Utilizamos aqui a taxa de crimes contra a propriedade e contra a pessoa (ambas por 100 mil habitantes, logaritmizadas), a taxa de desemprego formal (\%), e controles (\% de população jovem com idade entre 18 e 24 anos, taxa de urbanização \%, gasto em segurança pública \%) tal como proposto por Mocan \& Bali (2007). Os dados são municipais e cobrem o período entre 2000 e 2007. 


\section{RESULTADOS}

Os resultados das regressões são apresentados na Tabela 1. Na coluna I são apresentados os resultados estimados para a equação dos crimes contra a propriedade enquanto na coluna II temos aqueles para os crimes contra a pessoa. Vale ressaltar que, em ambos os casos, os testes de Sargan e de correlação serial não rejeitam a hipótese nula de especificação adequada dos modelos.

TABELA 1 - IMPACTO DE RECESSÕES E RECUPERAÇÕES SOBRE AS TAXAS DE CRIME (PAINEL MUNICIPAL MG)

\begin{tabular}{|c|c|c|}
\hline $\begin{array}{c}\text { GMM } \\
\begin{array}{c}\text { (instrumentos: primeiras defasa- } \\
\text { gens) }\end{array} \\
\end{array}$ & $\begin{array}{c}\text { Crimes contra a Propriedade } \\
\text { (estatística } \mathrm{z} \text { ) } \\
\text { I } \\
\end{array}$ & $\begin{array}{c}\text { Crimes contra a Pessoa } \\
\text { (estatística } \mathrm{z} \text { ) } \\
\text { II } \\
\end{array}$ \\
\hline Recessões $\left(\mathrm{LMO}^{+}\right)$ & $\begin{array}{r}0,00145^{* * *} \\
(1,80)\end{array}$ & $\begin{array}{r}-0,00017 \\
(-0,25)\end{array}$ \\
\hline Recuperações $\left(\mathrm{LMO}^{-}\right)$ & $\begin{array}{r}0,00180^{* *} \\
(2,27)\end{array}$ & $\begin{array}{r}-0,00024 \\
(-0,36)\end{array}$ \\
\hline População Idade 18_24 (\%) & $\begin{array}{r}-0,00006 \\
(-0,11)\end{array}$ & $\begin{array}{r}-0,00040^{* * *} \\
(-1,74)\end{array}$ \\
\hline Urbanização (\%) & $\begin{array}{r}0,00359 * * \\
(2,16)\end{array}$ & $\begin{array}{r}0.00157 \\
(1,01)\end{array}$ \\
\hline Gasto em Segurança (\%) & $\begin{array}{r}-0,00681 \\
(1,50)\end{array}$ & $\begin{array}{r}0,00557 \\
(1,28)\end{array}$ \\
\hline VDD & $\begin{array}{r}0,10412^{*} \\
(3,38)\end{array}$ & $\begin{array}{r}0,01323^{*} \\
(3,00)\end{array}$ \\
\hline Constante & $\begin{array}{r}0,06549 * \\
(11,80)\end{array}$ & $\begin{array}{r}0,01323^{*} \\
(3,00)\end{array}$ \\
\hline Teste Sargan (p-valor) & 0,0000 & 0,0213 \\
\hline $\begin{array}{l}\text { Teste Correlação Serial } 1^{a} \text { ordem } \\
\text { (p-valor) }\end{array}$ & 0,0000 & 0,0000 \\
\hline No. Observações & 3192 & 3731 \\
\hline
\end{tabular}

NOTAS: VDD: Variável dependente defasada.

* Se variável for significativa a $1 \%$, ** se variável for significativa a $5 \%$ e $* * *$ se variável for significativa a $10 \%$.

Podemos notar (coluna I) que, no caso dos crimes contra a propriedade, uma elevação de 1\% no desemprego municipal está associada a um aumento de $0,145 \%$ na taxa de crimes contra a propriedade (efeito significativo ao nível de 10\%). Por outro lado, uma redução de 1\% na taxa de desemprego levaria a uma redução na taxa de crimes contra a propriedade de $0,18 \%$ (efeito significativo ao nível de 5\%). Ou seja, de fato, o efeito de alterações nas condições do mercado de trabalho é assimétrico sobre a taxa de crime contra o patrimônio. Interessante notar que, para o caso dos municípios mineiros, os resultados contrariam o efeito de histerese 
tradicional suposto por Mocan, Billups \& Overland (2005). Os resultados encontrados para os crimes contra a pessoa sugerem que não há nenhuma reação da taxa de crime a mudanças na taxa de desemprego, pois ambos os coeficientes não são estatisticamente significativos $\left(\mathrm{LMO}^{+}\right.$ e $\left.\mathrm{LMO}^{-}\right)$.

Estes resultados podem ser explicados se considerarmos que crimes contra o patrimônio possuem um valor líquido esperado maior - já que geralmente resulta de um planejamento mais cuidadoso - relativamente aos crimes contra as pessoas. Além disso, a motivação econômica para crimes contra o patrimônio é mais clara do que nos crimes contras as pessoas.

Vale comentar sobre a magnitude da inércia criminal. O coeficiente estimado foi de 0,10412 e 0,09183 para os casos dos crimes contra a propriedade e contra a pessoa, respectivamente (ambos os parâmetros significativos ao nível de 1\%). Estes resultados sugerem que as elasticidades de longo prazo de todas as variáveis (significativas) dos modelos seriam 11,6 (crimes contra a propriedade) e 10,1\% (crimes contra a pessoa) maiores que as elasticidades de curto prazo. Do ponto de vista da persistência dos choques, isso implica que o tempo necessário para que o sistema percorra metade do caminho até o eventual equilíbrio em resposta a um choque é de cerca de 0,30 ano.

\section{CONCLUSÃO}

Evidências sobre a economia do crime mostram que o ambiente socioeconômico é um importante determinante das atividades criminosas. A maneira como se dá esta influência é alvo de várias hipóteses na literatura.

Neste artigo, toma-se como base a hipótese de que existem assimetrias na criminalidade conforme as fases do ciclo econômico segundo Mocan \& Bali (2007), porém com um método de estimação mais adequado, considerando-se possíveis problemas de endogeneidade e também a necessidade de inclusão de efeitos fixos (painel dinâmico).

Considerou-se dois tipos de crimes: contra a pessoa e contra o patrimônio, em um painel de municípios mineiros no período 2000-2007. Embora a literatura use o modelo beckeriano como base teórica para a análise empírica de ambos, parece-nos mais correto que a motivação econômica seja mais presente em crimes contra o patrimônio.

Os resultados indicam a existência de assimetria no ciclo econômico apenas no caso deste último tipo de crime, de forma que, aumentos no desemprego municipal geram um aumento no crime municipal menor que as quedas no mesmo quando da redução do desemprego. Em outras palavras, ao contrário do encontrado por Mocan \& Bali (2007), para nossa amostra, criminosos envolvidos em crimes contra o patrimônio têm mais facilidade a voltar ao mercado 
de trabalho legal ${ }^{3}$.

Adicionalmente, encontra-se inércia nas taxas de crime em ambas as regressões, o que sugere uma persistência temporal compatível com a literatura (e estimada em torno de $10 \%)^{4}$. Neste sentido, as elasticidades de longo prazo das variáveis independentes são também estimadas em $10 \%$ acima de seus valores de curto prazo.

Em resumo, e como apontado pela literatura, embora políticas públicas de combate ao crime sejam essenciais e importantes, há evidências de que o crescimento econômico, por si só, tenha um efeito-substituição sobre crimes mais diretamente ligados às tentativas de enriquecimento ilícito.

\section{REFERÊNCIAS}

ARELLANO, M. and S. BOND. Some Tests of Specification for Panel Data: Montecarlo Evidence and an Application to Employment Equations. Review of Economic Studies, v. 58(2), p. 277-297.

ARELLANO, M. and O. BOVER. Another Look at the Instrumental-Variable Estimation of Error-Components Models. Journal of Econometrics, v. 68(1), p. 29-52.

BECKER, G.S. Crime and Punishment: An Economic Approach. Journal of Political Economy, v.76, p. 169-217.

FAJNZYLBER, P.; ARAUJO JUNIOR, A. F. Violência e Criminalidade. In: Lisboa, M.B.; Menezes-Filho, N.A. (Org.). Microeconomia e Sociedade no Brasil. Editora Contra Capa/FGV.

HOLTZ-EAKIN, D.; W. NEWEY, and H. S. ROSEN. Estimating Vector Autoregressions with Panel Data. Econometrica, v.56, p.1371-1395.

MOCAN, BALI. Asymmetric Crime Cycles, with Turan Bali. Forthcoming in The Review of Economics and Statistics.

MOCAN, BILLUPS \& OVERLAND. A Dynamic Model of Differential Human Capital and Criminal Activity. Economica, November 2005, v. 72(288), p. 655-81.

SANTOS, M.J. dos. Dinâmica Temporal da Criminalidade: Mais Evidências Sobre o Efeito Inercial nas Taxas de Crimes Letais nos Estados Brasileiros. Revista Economia, v. 10(1), p. 169-164.

3 Não dispomos de bases de dados que nos mostrem quantos indivíduos envolvidos em crimes reportados na base de dados do IRMS, são, efetivamente, capturados. Também não temos estatísticas de reconversão dos mesmos para o mercado de trabalho legal. Talvez o resultado encontrado seja uma evidência de que, dentre todos os criminosos não capturados, os que possuem maior facilidade de voltarem ao mercado de trabalho sejam os menos envolvidos em crimes com motivações relativamente menos econômicas como estupro, rixas.

4 Ver, por exemplo, a resenha de Santos (2009). 